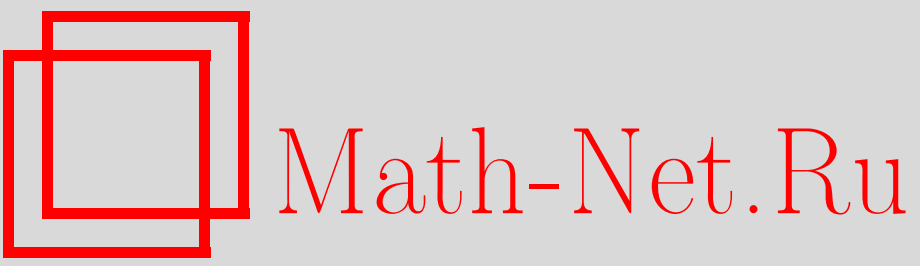

А. П. Нерсесян, Гамильтонов формализм для частиц с обобщенной жесткостью, ТМФ, 1998, том 117, номер 1, 130-139

DOI: https://doi.org/10.4213/tmf922

Использование Общероссийского математического портала Math-Net.Ru подразумевает, что вы прочитали и согласны с пользовательским соглашением

http://www.mathnet.ru/rus/agreement

Параметры загрузки:

IP : 54.196 .121 .252

26 апреля 2023 г., 18:24:26 
ТЕОРЕТИЧЕСКАЯ

И МАТЕМАТИЧЕСКАЯ

ФИЗИКА

Том 117, № 1

октябрь, 1998

(C) 1998 г.

А.П. Нерсесян ${ }^{*}$

\section{ГАМИЛЬТОНОВ ФОРМАЛИЗМ ДЛЯ ЧАСТИЦ С ОБОБШЕННОЙ ЖЕСТКОСТЬЮ}

Разработан гамильтонов формализм для механических систем с репараметризационно-инвариантными лагранжианами, зависящими от внешних кривизн мировой линии. Построены полные системы связей для лагранжианов с квадратичной зависимостью от внешних кривизн, лагранжианов, пропорциональных произвольной внешней кривизне, и лагранжианов, линейно зависящих от первых двух кривизн.

\section{1. ВВЕДЕНИЕ}

Как известно, кривая в $D$-мерном пространстве определяется $(D-1)$ репараметризационными инвариантами (внешними кривизнами) $\tilde{k}_{1}, \ldots, \tilde{k}_{D-1}$, являюшимися функциями натурального параметра кривой $\tilde{s}$ [1]. Поэтому репараметризационно-инвариантное действие можно задать лагранжианом, являюшимся функцией внешних кривизн мировой линии:

$$
\mathcal{S}=\int F\left(\tilde{k}_{1}, \ldots, \tilde{k}_{N}\right) d \tilde{s}, \quad 0 \leqslant N \leqslant D-1 .
$$

Такие системы мы будем называть моделями частиц с обобщенной жесткостью.

Механические лагранжианы, зависяшие от первой и второй кривизн мировой линии, начали относительно интенсивно исследоваться в конще восьмидесятых годов как простые модели жестких струн и $(2+1)$-мерных теорий поля с членом Черна-Саймонса [2]. Вскоре стало ясно [3-5], что данные системы представляют самостоятельный интерес, прежде всего при изучении спиновых частиц. Так, при $D=(2+1), F=c_{0}+c_{1} \tilde{k}_{1}+c_{2} \tilde{k}_{2}$, $c_{0} \neq 0$ таким системам отвечает массивный релятивистский анион [3], при $D=(3+1)$, $F=c_{0}+c_{1} \tilde{k}_{1}, c_{0} \neq 0$-массивный релятивистский бозон [4], а при $D=(3+1), F=c \tilde{k}_{1}-$ безмассовая частища произвольной (целой и полуцелой) спиральности [5]. Заметим, что при $F=c_{0}+k_{1}^{2}$ мы имеем эффективное действие релятивистского кинка в поле солитона [6].

Недавно было показано [7], что система с $F=c \tilde{k}_{1}$ обладает калибровочной $W_{3}$-симметрией. В работе [8] указывается на наличие в системе с лагранжианом $F=c \tilde{k}_{N}$ $(N+1)$ калибровочных симметрий, образуюших предположительно $W_{N+2}$-алгебру.

*Объединенный институт ядерных исследований, Лаборатория теоретической физики им. Н. Н. Боголюбова, Дубна, Московская обл., Россия. E-mail: nerses@thsun1.jinr.ru 
Ответ на вопросы: какие (изо)спиновые частицы описываются моделями с обобшенной жесткостью и какими калибровочными $W$-симметриями могут обладать эти модели, предполагает знание размерности и структуры фазовых пространств рассматриваемых моделей, генераторов калибровочных симметрий и их последуюшее квантование.

Для этого прежде всего требуется построить гамильтонов формализм для систем (1.1). Заметим, что лагранжианы моделей частиц с обобщенной жесткостью зависят от производных $(N+1)$-го порядка, т.к. внешние кривизны определяются выражениями

$$
\tilde{k}_{I}(\tilde{s})=\frac{\sqrt{\operatorname{det} \widehat{g}_{I+1} \operatorname{det} \widehat{g}_{I-1}}}{\operatorname{det} \widehat{g}_{I}}, \quad\left(g_{I}\right)_{i j} \equiv \mathbf{x}_{(i)} \mathbf{x}_{(j)}, \quad i, j=1, \ldots, I,
$$

где $\mathbf{x}_{(i)} \equiv d^{i} \mathbf{x}(\tilde{s}) /(d \tilde{s})^{i}$. Потому вначале нужно заменить исходный лагранжиан эквивалентным лагранжианом второго (первого) порядка и лишь после этого перейти к гамильтонову формализму в $2 D(N+1)$-мерном фазовом пространстве.

Однако при переходе к гамильтонову описанию большинство авторов игнорирует инвариантные свойства лагранжианов, определяемые их зависимостью от внешних кривизн. В результате даже построение системы первичных связей предполагает проведение утомительных бесструктурных пребразований. Так, в уже упомянутой работе [8] удалось построить сушественно нелинейную полную систему связей лишь для $F=c \tilde{k}_{2}$.

В данной работе предлагается более "геометричный" способ построения гамильтонова формализма для моделей частиц с обобщенной жесткостью, который основан на формулах Френе для подвижного репера, определяюших внешние кривизны.

Полученная таким образом гамильтонова система задается с помощью координат исходного пространства $\mathbf{x}$, компонент подвижного репера $\mathbf{e}_{i}$ и сопряженных им импульсов $\mathbf{p}$ и $\mathbf{p}_{i}$, где $i=1, \ldots, N$. Лагранжевы множители при первичных связях имеют смысл внешних кривизн мировой линии.

Эффективность предлагаемых построений демонстрируется определением полных наборов связей и гамильтонианов для моделей со следуюшими лагранжианами:

а) система

$$
F=\frac{1}{2} \sum_{i}^{N} b_{i} \tilde{k}_{i}^{2}+\sum_{i=1} c_{i} \tilde{k}_{i}+c_{0}, \quad b_{1} b_{2} \ldots b_{N} \neq 0,
$$

характеризуется наименьшим вырождением и отсутствием вторичных связей;

б) система

$$
F=c \tilde{k}_{N}, \quad \forall D \quad N<D
$$

характеризуется максимальным (при данном $N)$ вырождением и $(N+1)$ калибровочными степенями свободы; все возникающие в ней связи квадратичны.

Мы покажем, что системы с лагранжианами, линейно зависяшими от внешних кривизн, обладают максимально возможным набором (квадратичных) первичных связей. Наличие в лагранжиане кривизн $k_{a}, a<N$, сушественно определяет набор вторичных связей и уменьшает калибровочную инвариантность лагранжиана. Поэтому мы приводим для иллюстрации полные наборы связей для хорошо изученных моделей с лагранжианами $F=c_{0}+c_{1} \tilde{k}_{1}$ и $F=c_{0}+c_{1} \tilde{k}_{1}+c_{2} \tilde{k}_{2}$.

5 Теоретическая и математическая физика, т. 117, № 1, 1998 г. 
Везде далее мы полагаем для простоты сигнатуру пространства $R^{D}$ евклидовой, что не должно привести к недоразумениям при переходе к псевдоевклидову пространству.

Мы будем пользоваться группами индексов

$$
i, j, k=1, \ldots N ; \quad a, b, c, d=1, \ldots(N-1) ; \quad \alpha, \beta=1, \ldots,(N-2)
$$

и обозначениями

$$
\begin{gathered}
F_{, i} \equiv \frac{\partial F}{\partial \tilde{k}_{i}}, \quad F_{i j} \equiv \frac{\partial^{2} F}{\partial \tilde{k}_{i} \partial \tilde{k}_{j}} \\
\widetilde{\phi}_{0 . i}=\mathbf{p e}_{i}, \quad \widetilde{\phi}_{i . j}=\mathbf{p}_{i} \mathbf{e}_{j}-\mathbf{p}_{j} \mathbf{e}_{i}, \quad \widetilde{\Phi}_{00}=\mathbf{p} \widehat{L} \mathbf{p}, \quad \widetilde{\Phi}_{0 i}=\mathbf{p} \widehat{L} \mathbf{p}_{i}, \quad \widetilde{\Phi}_{i j}=\mathbf{p}_{i} \widehat{L} \mathbf{p}_{j}
\end{gathered}
$$

где

$$
\widehat{L}=\widehat{I}-\sum_{i=1}^{N} \mathbf{e}_{i} \otimes \mathbf{e}_{i} ; \quad \forall \mathbf{a} \quad \mathbf{b}: \mathbf{a} \equiv a^{A}, \quad \mathbf{b} \equiv a^{A}, \quad \mathbf{a b}=\sum_{A=1}^{D} a^{A} b^{A}
$$

\section{2. ФОРМУЛЫ ФРЕНЕ И ОБОБЩЕННОЕ ПРЕОБРАЗОВАНИЕ ЛЕЖАНДРА}

Рассмотрим гамильтонову формулировку системы (1.1).

Представим действие (1.1) в виде

$$
\mathcal{S}=\int F\left(\frac{k_{1}}{s}, \ldots, \frac{k_{N}}{s}\right) s d \tau, \quad \text { где } s \equiv\left|\frac{d \mathbf{x}}{d \tau}\right|, \quad k_{i} \equiv s \tilde{k}_{i} .
$$

Пусть $\left\{\mathbf{e}_{\mu}\right\}$ задает подвижный репер траектории системы:

$$
\mathbf{e}_{\mu} \mathbf{e}_{\nu}=\delta_{\mu \nu}, \quad \dot{\mathbf{x}}=s \mathbf{e}_{1}, \quad \mu=1, \ldots, D
$$

В терминах подвижного репера внешние кривизны определяются уравнениями Френе

$$
\dot{\mathbf{e}}_{\mu}=k_{\mu} \mathbf{e}_{\mu+1}-k_{\mu-1} \mathbf{e}_{\mu-1}, \quad \mathbf{e}_{0}=\mathbf{e}_{D+1}=0
$$

Отсюда легко найти явные выражения для внешних кривизн

$$
k_{\mu-1}=\dot{\mathbf{e}}_{\mu-1} \mathbf{e}_{\mu}, \quad k_{\mu}^{2}=\dot{\mathbf{e}}_{\mu}^{2}-k_{\mu-1}^{2} .
$$

Заметим, что $k_{\mu} \geqslant 0$ при $\mu=1, \ldots,(D-2)$, тогда как $k_{D-1}$ ("кручение") может принимать как положительные, так и отрицательные значения. Если $k_{I} \neq 0$, то $k_{\mu} \neq 0$ при $\mu=1,2, \ldots, I-1$. Обратно, если $k_{I}=0$, то $k_{\mu}=0$ при $\mu=I+1, \ldots, D-1[1]$.

Учитывая выражения (2.2)- (2.4), можно заменить первоначальный лагранжиан следующим лагранжианом второго порядка:

$$
\begin{aligned}
L= & F\left(\frac{k_{1}}{s}, \ldots, \frac{k_{N}}{s}\right) s+\mathbf{p}\left(\dot{\mathbf{x}}-s \mathbf{e}_{1}\right)+\sum_{a} \mathbf{p}_{a}\left(\dot{\mathbf{e}}_{a}-k_{a} \mathbf{e}_{a+1}+k_{a-1} \mathbf{e}_{a-1}\right)- \\
& -\sum_{i, j} d^{i j}\left(\mathbf{e}_{i} \mathbf{e}_{j}-\delta_{i j}\right)-F_{, N}\left(k_{N}-\left(\dot{\mathbf{e}}_{N}^{2}-k_{N-1}^{2}\right)^{1 / 2}\right)
\end{aligned}
$$


где $s, k_{i}, d^{i j}, \mathbf{p}_{a}, \mathbf{e}_{i}$ играют роль независимых переменных.

Теперь совершим преобразование Лежандра для лагранжиана (2.5). Переменные $\mathbf{p}_{a}$ играют роль импульсов, сопряженных $\mathbf{e}_{a}$, а импульсы, сопряженные $\left(s, k_{a}, d_{i j}\right)$, приводят к тривиальным связям

$$
p^{s} \approx 0, \quad p^{a} \approx 0, \quad p^{i j} \approx 0
$$

Полагая $k_{N} \neq 0, F_{, N} \neq 0$ получаем, что импульс, сопряженный $\mathbf{e}_{N}$, имеет вид

$$
\mathbf{p}_{N}=F_{, N}\left(\dot{\mathbf{e}}_{N}^{2}-k_{N-1}^{2}\right)^{-1 / 2} \dot{\mathbf{e}}_{N}
$$

Отсюда с учетом формул Френе получаем следующие связи:

$$
\begin{aligned}
& \chi_{N . N} \equiv \mathbf{p}_{N} \mathbf{e}_{N} \approx 0, \quad \chi_{N . \alpha}=\mathbf{p}_{N} \mathbf{e}_{\alpha} \approx 0, \\
& \Phi_{N . N}=\mathbf{p}_{N}^{2}-\left(\mathbf{p}_{N} \mathbf{e}_{N-1}\right)^{2}-F_{, N}^{2} \approx \widetilde{\Phi}_{N . N}-F_{N}^{2} \approx 0 .
\end{aligned}
$$

Совершив преобразование Лежандра, получим с учетом этих связей следующий тотальный гамильтониан

$$
\mathcal{H}_{\mathrm{T}}=\mathcal{H}+\lambda^{(s)} p_{s}+\lambda^{(k) a} p^{a}+\lambda_{(d) i j} p^{i j}
$$

где

$$
\begin{aligned}
& \mathcal{H}=s \phi_{0.1}+\sum_{a} k_{a} \phi_{a . a+1}+\lambda \Phi_{N . N}+\sum_{i, j} d^{i j} u_{i j}+\sum_{\alpha} \lambda_{\alpha} \chi_{N . \alpha}+\lambda_{N} \chi_{N . N}, \\
& u_{i j} \equiv \mathbf{e}_{i} \mathbf{e}_{j}-\delta_{i j}, \quad \phi_{a . a+1} \equiv \widetilde{\phi}_{a . a+1}-F_{, a}, \quad \phi_{0.1} \equiv \widetilde{\phi}_{0.1}+\sum_{i} \tilde{k}_{i} F_{, i}-F
\end{aligned}
$$

а $\lambda, \lambda_{\alpha}, \lambda_{N}-$ лагранжевы множители.

Стабилизация (2.6) порождает вторичные связи

$$
\begin{aligned}
& u_{i j} \approx 0, \quad s \phi_{0.1}+\sum_{a} k_{a} \phi_{a . a+1} \approx 0 \Rightarrow \mathcal{H} \approx 0, \\
& s \phi_{a . a+1}=-F_{, N a}\left(k_{N}-2 \lambda F_{N}\right), \quad\left(k_{N}-2 \lambda F_{N}\right) F_{, N N} \approx 0 .
\end{aligned}
$$

Теперь, исключив связи (2.6), мы получим гамильтонову систему, описываемую гамильтонианом (2.11) и симплектической структурой

$$
\omega_{N}=d \mathbf{p} \wedge d \mathbf{x}+\sum_{i=1}^{N} d \mathbf{p}_{i} \wedge d \mathbf{e}_{i}
$$

Выражения (2.8), (2.13) задают в редуцированной системе первичные связи. Выражения $(2.14),(2.9)$ либо определяют переменные $k_{a}, k_{N}$ как функции от $\widetilde{\phi}_{0.1}, \widetilde{\phi}_{a . a+1}$, либо задают связи, при которых переменные $k_{a}, k_{N}$ играют роль лагранжевых множителей. Число возникаюших связей равно корангу гессиана $F_{i j}$. 
Заметим, что функции (1.2) образуют замкнутую относительно скобок Пуассона (квадратичную) алгебру и удовлетворяют уравнениям

$$
\left\{\widetilde{\phi}_{\ldots}, u_{\ldots}\right\} \approx\left\{\widetilde{\Phi}_{\ldots}, u_{\ldots}\right\} \approx 0, \quad\left\{\chi_{N i}, u_{j k}\right\} \approx \delta_{N j} \delta_{i k}
$$

Отсюда видим, что:

1) все вторичные связи зависят от функций (1.2);

2 ) связи $u_{N . N}, u_{N . \alpha}$ и $\chi_{N \alpha}, \chi_{N . N}$ являются взаимно-сопряженными связями второго рода, причем

$$
\lambda_{N \alpha}=\lambda_{N}=0
$$

$3)$ связи $u_{N . N-1}, u_{a . b}-$ связи первого рода, а их стабилизация не порождает вторичных связей.

Следовательно, размерность фазового пространства системы удовлетворяет неравенству

$$
(2 D-3 N-2)(N+1) \leqslant D_{\text {red }} \leqslant(2 D-N)(N+1)-2 .
$$

Принимая во внимание, что первичные связи первого рода порождают калибровочные преобразования [9], а преобразования лагранжиана, вызываемые первичными связями $u_{N . N-1}, u_{a . b}$, тривиальны, заключаем, что число нетривиальных калибровочных преобразований лагранжиана не превышает $\operatorname{corank}\left(F_{i j}\right)+1$.

Удобно зафиксировать величины $d_{N . N-1}$ и $d_{a . b}$, наложив калибровочные условия

$$
\chi_{N . N-1} \equiv \mathbf{p}_{N} \mathbf{e}_{N-1} \approx 0, \quad \chi_{a . a-\kappa} \equiv \mathbf{p}_{a} \mathbf{e}_{a-\kappa} \approx 0, \quad \kappa=0, \ldots, a-1,
$$

удовлетворяющие равенствам

$$
\begin{aligned}
& \left\{\chi_{i . j}, u_{k l}\right\} \approx \delta_{i\{k} \delta_{l\} j}, \quad\left\{\chi_{i . j}, \widetilde{\Phi}_{N N}\right\} \approx 2 \delta_{N . i} \delta_{i j} \widetilde{\Phi}_{N N} \\
& \left\{\chi_{i . j}, \widetilde{\phi}_{a . a+1}\right\} \approx \delta_{i j}\left(\delta_{i . a}-\delta_{i . a+1}\right) \widetilde{\phi}_{a . a+1} .
\end{aligned}
$$

ПримеР. В максимально невырожденном случае, когда $\operatorname{det} F_{i j} \neq 0$, лагранжиан системы обладает лиш репараметризационной инвариантностью, а размерность фазового пространства системы $D_{\max }=(2 D-N)(N+1)-2$.

Рассмотрим простейший пример такой системы:

$$
F=\frac{1}{2} \sum_{i}^{N} b_{i} \tilde{k}_{i}^{2}+\sum_{i=1} c_{i} \tilde{k}_{i}+c_{0}, \quad b_{1} b_{2} \ldots b_{N} \neq 0 .
$$

Разрешив связи (2.9), (2.14), получаем, что

$$
\tilde{k}_{a}=\frac{\widetilde{\phi}_{a . a+1}-c_{a}}{b_{a}}, \quad\left(b_{N} \tilde{k}_{N}+c_{N}\right)^{2}=\widetilde{\Phi}_{N . N}
$$

откуда

$$
\mathcal{H}=s \phi_{0.1}+d_{i j} u_{i j}, \quad \phi_{0.1}=\widetilde{\phi}_{0.1}+\frac{1}{2} \sum_{i} b_{i} \tilde{k}_{i}^{2}-c_{0} .
$$

Система имеет лишь первичные связи $\phi_{0.1} \approx 0, u_{i j} \approx 0, \chi_{N . N} \approx 0, \chi_{N . \alpha} \approx 0$. Стабилизация связей (2.8) и калибровочных условий (2.18) приводит к фиксации матрицы $d_{i j}$, значения которой мы не выписываем за ненадобностью. 


\section{3. ЛАГРАНЖИАНЫ, ЛИНЕЙНО ЗАВИСЯЩИЕ ОТ КРИВИЗН}

Как видно из соотношений (2.14), максимально вырожденными являются системы с лагранжианами, линейно зависящими от внешних кривизн,

$$
F=c_{0}+\sum_{i=1}^{N} c_{i} \tilde{k}_{i}
$$

Такие системы имеют следующий (максимальный) набор первичных связей:

$$
\begin{aligned}
& \phi_{0.1}=\mathbf{p e}_{1}-c_{0} \approx 0, \\
& \phi_{a . a+1}=\mathbf{p}_{a} \mathbf{e}_{a+1}-\mathbf{p}_{a+1} \mathbf{e}_{a}-c_{a} \approx 0, \\
& \Phi_{N . N}=\mathbf{p}_{N} \widehat{L} \mathbf{p}_{N}-c_{N}^{2} \approx 0, \\
& \chi_{N . N} \equiv \mathbf{p}_{N} \mathbf{e}_{N} \approx 0, \quad \chi_{N . \alpha}=\mathbf{p}_{N} \mathbf{e}_{\alpha} \approx 0, \\
& u_{i j}=\mathbf{e}_{i} \mathbf{e}_{j}-\delta_{i j} \approx 0,
\end{aligned}
$$

и гамильтониан

$$
\mathcal{H}=s \phi_{0.1}+\sum_{a=1}^{N-1} k_{a} \phi_{a . a+1}+\lambda \Phi_{N . N}+\sum_{i, j=1}^{N} d^{i j} u_{i j} .
$$

Из уравнений движения для репера $\mathbf{e}_{N}$ видим, что $2 c_{N} \lambda=k_{N}=s \tilde{k}_{N}$.

Таким образом, в гамильтоновом формализме метрические инварианты траектории $s, k_{i}$ играют роль лагранжевых множителей.

Поскольку при преобразовании Лежандра требовалось вьполнение условия $k_{N} \neq 0$, то при стабилизации первичных связей системы мы должны полагать $k_{a} \neq 0, \lambda \neq 0$.

Наложим калибровочные условия (2.18), в результате чего все первичные связи станут квадратичными. Условия (2.18) вместе со связями (2.8) дают следуюшую фиксацию калибровки:

$$
d_{i . j}=\delta_{i j}\left(k_{i} c_{i}-k_{i-1} c_{i-1}-s \delta_{1 . i} c_{0}\right) .
$$

Стабилизация остальных первичных связей порождает вторичные связи первого этапа

$$
\widetilde{\phi}_{0.2} \approx 0, \quad \widetilde{\phi}_{\alpha . \alpha+2} \approx 0, \quad \widetilde{\Phi}_{N . N-1} \approx 0 .
$$

Заметим, что эволюция функций (1.2) определяется следуюшими выражениями:

$$
\begin{aligned}
& \dot{\Phi}_{00}=-4 \lambda \Phi_{0 N} \phi_{0 N}, \\
& \dot{\phi}_{0 i}=-k_{i-1} \phi_{0 . i-1}+k_{i} \phi_{0 . i+1}+2 \lambda \delta_{i N} \Phi_{0 N}, \\
& \dot{\Phi}_{0 i}=-s \delta_{1 i} \Phi_{00}-k_{i-1} \Phi_{0 . i-1}+k_{i} \Phi_{0 . i+1}-2 \lambda \Phi_{N\{i} \phi_{0\} N}, \\
& \dot{\phi}_{i j}=-s \delta_{1[i} \phi_{j] 0}-k_{i-1} \phi_{i-1 . j}+k_{i} \phi_{i+1 . j}-k_{j-1} \phi_{i . j-1}+k_{j} \phi_{i . j+1}+2 \lambda \delta_{N[i} \Phi_{j] N}, \\
& \dot{\Phi}_{i j}=-s \delta_{1\{i} \Phi_{j\} 0}-k_{i-1} \Phi_{i-1 . j}+k_{i} \Phi_{i+1 . j}-k_{j-1} \Phi_{j-1 . i}+k_{j} \Phi_{j+1 . i}-2 \lambda \Phi_{N\{i} \phi_{j\} N} .
\end{aligned}
$$


Отсюда видим, что дальнейшее проведение процедуры Дирака существенно зависит от значений констант $c_{i}$.

Так, если лагранжиан (3.1) конформно-инвариантен $\left(c_{0}=0\right)$, то стабилизация связи $\widetilde{\phi}_{0.1} \approx 0$ приводит к следующей цепочке связей первого рода:

$$
\widetilde{\phi}_{0 . i}=\mathbf{p e}_{i} \approx 0, \quad \widetilde{\Phi}_{0 . i} \approx \mathbf{p p}_{i} \approx 0, \quad \widetilde{\Phi}_{0.0} \approx \mathbf{p}^{2} \approx 0,
$$

что отвечает (в псевдоевклидовом пространстве) безмассовому случаю ${ }^{1)}$.

Стабилизация остальных связей уже не затрагивает пространственный импульс системы, лишь специфицируя ее "внутреннее" пространство.

Нетрудно увидеть, что максимальным набором связей (и калибровочных симметрий) обладают системы (3.1) с $c_{0}=c_{1}=\cdots=c_{N-1}=0, c_{N} \equiv c \neq 0$. Для таких систем проведение процедуры Дирака порождает дополнительно к связям (3.6) следующий набор связей:

$$
\widetilde{\phi}_{i, j} \approx 0, \quad \widetilde{\Phi}_{i . j}-c^{2} \delta_{i j} \approx 0 .
$$

Все они являются связями первого рода.

Наложим калибровочные условия (2.18) и введем комплексные координаты

$$
\mathbf{z}_{i}=\frac{\mathbf{p}_{i}+i c \mathbf{e}_{i}}{\sqrt{2}}, \quad \omega_{2}=d \mathbf{p} \wedge d \mathbf{x}+\frac{i}{c} \sum_{i} d \mathbf{z}_{i} \wedge d \overline{\mathbf{z}}_{i}
$$

В этих координатах гамильтониан системы задается выражением

$$
\mathcal{H}=\frac{s}{2 c}\left[i \sqrt{2} \mathbf{p}\left(\overline{\mathbf{z}}_{1}-\mathbf{z}_{1}\right)+i \sum_{a=1}^{N-1} \tilde{k}_{a}\left(\mathbf{z}_{a} \overline{\mathbf{z}}_{a+1}-\mathbf{z}_{a+1} \overline{\mathbf{z}}_{a}\right)+\tilde{k}_{N}\left(\mathbf{z}_{N} \overline{\mathbf{z}}_{N}-c^{2}\right)\right] .
$$

Связи (3.6), (3.7), (2.8) и калибровочные условия (2.18) принимают вид

$$
\Phi_{i \bar{j}}^{0} \equiv \mathbf{z}_{i} \overline{\mathbf{z}}_{j}-c^{2} \delta_{i j} \approx 0, \quad \Phi_{i}^{+} \equiv \mathbf{p} \mathbf{z}_{i} \approx 0, \quad \Phi_{0}=\mathbf{p}^{2} \approx 0, \quad U_{i j}^{+}=\frac{1}{2} \mathbf{z}_{i} \mathbf{z}_{j} \approx 0
$$

Введенные с помощью соотношений (3.10) величины образуют алгебру

$$
\begin{aligned}
& \left\{\Phi_{i \bar{j}}, \Phi_{k \bar{l}}\right\}=i c\left(\delta_{i \bar{l}} \Phi_{k \bar{j}}-\delta_{k \bar{j}} \Phi_{i \bar{l}}\right), \quad\left\{\Phi_{i \bar{j}}, \Phi_{k}^{+}\right\}=-i c \delta_{k \bar{j}} \Phi_{i}^{+}, \\
& \left\{\Phi_{i \bar{j}}, U_{k l}^{+}\right\}=-i c\left(\delta_{k \bar{j}} U_{i l}^{+}+\delta_{l \bar{j}} U_{i k}^{+}\right), \quad\left\{\Phi_{i}^{+}, U_{\bar{j} \bar{k}}^{-}\right\}=\frac{1}{2} i c \delta_{i\{\bar{j}} \Phi_{\bar{k}\}}^{-}, \\
& \left\{\Phi_{i}^{+}, \Phi_{\bar{j}}^{-}\right\}=i c \delta_{i \bar{j}} \Phi_{0}, \quad\left\{\Phi, U_{i j}^{+}\right\}=\left\{\Phi, \Phi_{i \bar{j}}\right\}=\left\{\Phi, \Phi_{i}^{+}\right\}=0, \\
& \left\{\Phi_{i}^{+}, U_{j k}^{+}\right\}=\left\{\Phi_{i}^{+}, \Phi_{j}^{+}\right\}=\left\{U_{i j}^{+}, U_{k l}^{+}\right\}=0, \\
& \left\{U_{i j}^{+}, U_{\bar{k} \bar{l}}^{-}\right\}=\frac{1}{4} i c \delta_{\{i \cdot\{\bar{k}} \Phi_{j\} \bar{l}\}}+\frac{1}{2} i c^{3}\left(\delta_{i \bar{k}} \delta_{j \bar{l}}+\delta_{i \bar{l}} \delta_{j \bar{k}}\right),
\end{aligned}
$$

\footnotetext{
1) Полный импульс системы $\mathbf{P}$ и генераторы ее вращений $\mathbf{M}^{(2)}$ определяются выражениями
}

$$
P^{A}=p^{A}, \quad M^{(2) A B}=p^{[A} x^{B]}+\sum_{i=1}^{N} p_{i}^{[A} e_{i}^{B]} .
$$


где $\Phi_{i}^{-}=\bar{\Phi}_{i}^{+}, U_{i j}^{-}=\bar{U}_{i j}^{+}$.

Таким образом, $U_{i j}^{ \pm}$являются связями второго рода, а остальные связи - первого рода. Размерность фазового пространства

$$
D_{\min }=(2 D-3 N-2)(N+1),
$$

система обладает $(N+1)$ калибровочными степенями свободы.

Из (3.11) видно, что если $D=4$, то динамика нетривиальна лишь при $N=1$. При этом размерность фазового пространства системы совпадает с размерностью фазового пространства безмассовой частицы со спиральностью $c$ в $(3+1)$-мерном пространстве [3]. В таком пространстве можно “спиноризовать" связи (3.10) и провести ковариантное квантование системы [10].

Как видно из соотношений (3.10), сходную процедуру можно провести также при $N>1$ в $(5+1)-,(7+1)$ - и $(9+1)$-мерных пространствах.

Однако наиболее любопытным нам представляется совпадение построенной системы связей с системой $(N+1)$ осцилляторов, полученной дискретизацией струны $[11,12]$.

Рассмотрим случай системы (3.1) при $N=1, c_{0} \neq 0$. Имеются одна вторичная связь и одно условие на лагранжевы множители:

$$
\widetilde{\Phi}_{0.1} \approx 0, \quad s \widetilde{\Phi}_{00}+k_{1} c_{1} c_{0}=0
$$

Заметим, что $\dot{\widetilde{\Phi}}_{00}=0$, откуда получаем, что $p^{2}=c_{0}^{2}-c_{0} c_{1} \tilde{k}_{1}=$ const, т.е. траектория системы имеет постоянную кривизну. В псевдоевклидовом пространстве последнее равенство означает сохранение массы системы на заданной траектории движения.

В комплексных координатах (3.8), где $c \equiv c_{1}$, полный набор связей можно представить в виде одной вешественной и двух голоморфных связей

$$
\Phi=\mathbf{z} \overline{\mathbf{z}}-c_{1}^{2}, \quad U^{+}=\frac{\mathbf{z}^{2}}{2}, \quad \Phi^{+}=\mathbf{p} \mathbf{z}-\frac{i c_{0} c_{1}}{\sqrt{2}},
$$

образуюших алгебру

$$
\begin{array}{lll}
\left\{\Phi, \Phi_{+}\right\}=-i c_{1} \Phi_{+}+\frac{c_{1}^{2} c_{0}}{\sqrt{2}}, & \left\{\Phi, U^{+}\right\}=-2 i c_{1} U^{+}, & \left\{U^{+}, U^{-}\right\}=i c_{1} \Phi+i c_{1}^{3}, \\
\left\{\Phi^{+}, \Phi^{-}\right\}=i c_{1} \mathbf{p}^{2}, & \left\{\Phi_{+}, U_{-}\right\}=i c_{1} \Phi^{-}+\frac{c_{0} c_{1}^{2}}{\sqrt{2}}, & \left\{\Phi_{+}, U_{+}\right\}=0,
\end{array}
$$

где $\Phi_{-} \equiv \bar{\Phi}_{+}, U_{-} \equiv \bar{U}_{+}$.

Таким образом, при $c_{0} \neq 0, \quad N=1$ размерность фазового пространства $D_{\text {red }}=$ $2(2 D-3)$.

Система имеет единственную калибровочную степень свободы, задаваемую гамильтонианом

$$
\mathcal{H}_{1 . c_{0} \neq 0}=\frac{s}{2 c_{1}^{2} c_{0}}\left[2 i c_{1}^{2} c_{0} \mathbf{p}(\mathbf{z}-\overline{\mathbf{z}})+\left(c_{0}^{2}-\mathbf{p}^{2}\right)\left(\mathbf{z} \overline{\mathbf{z}}-c_{1}^{2}\right)\right] .
$$


При $c_{0}=0$ размерность фазового пространства $D_{\text {red }}=2(2 D-5)$, и система имеет две калибровочные степени свободы.

Теперь рассмотрим случай $N=2$ при произвольных константах $c_{0}, c_{1}, c_{2}$.

При $c_{0} \neq 0$ имеем две вторичные связи второго этапа, определяемые выражениями (3.5). Их стабилизация приводит к условиям

$$
c_{2} c_{0} k_{1}=k_{2} \widetilde{\Phi}_{0.2}, \quad s c_{0} c_{2} \widetilde{\Phi}_{0.2}=k_{2}\left(\Phi_{1.1} \widetilde{\Phi}_{0.2}-c_{2}^{2} c_{1} c_{0}\right)
$$

где $\Phi_{1.1} \equiv \widetilde{\Phi}_{1.1}-c_{2}^{2}, \widetilde{\Phi}_{0.2} \neq 0$. При $c_{1}=0$, второе условие принимает вид $s c_{0} c_{1}+$ $k_{2} \Phi_{1.1}=0$. Система имеет единственную калибровочную степень свободы, размерность фазового пространства $D_{\text {red }}=6(D-2)$.

Как и при $N=1$, имеем интеграл движения $\widetilde{\Phi}_{0.0}: \dot{\widetilde{\Phi}}_{0.0}=0$, отвечающий в псевдоевклидовом пространстве за сохранение массы на заданной траектории движения.

При $c_{0}=0, c_{1} \neq 0$ вторичные связи определяются выражениями $(3.6)$ и $\widetilde{\Phi}_{1.2} \approx 0$. Имеет место условие

$$
k_{2} c_{2} c_{1}+k_{1} \Phi_{1.1}=0
$$

при этом $\Phi_{1.1}$ является интегралом движения: $\dot{\Phi}_{1.1}=0$. Отсюда следует, что $\tilde{k}_{2} / \tilde{k}_{1}=$ const. Система имеет две калибровочные степени свободы, размерность ее фазового пространства $D_{\text {red }}=2(3 D-10)$.

При $c_{0}=c_{1}=0$ размерность фазового пространства системы $D_{\text {red }}=6(D-4)$, имеются три калибровочные степени свободы.

\section{4. ЗАКЛЮЧЕНИЕ}

Мы построили гамильтонов формализм для репараметризационно-инвариантных механических систем общего положения. В частности, мы показали, что размерность фазового пространства $D$-мерной системы с лагранжианом, зависяшим от первых $N$ внешних кривизн, удовлетворяет неравенству (2.17). Эта размерность максимальна для лагранжианов, являюшихся невырожденной квадратичной функцией внешних кривизн, и минимальна для лангранжианов, пропорциональных единственной $N$-й внешней кривизне.

В первом случае лагранжианы имеют единственную калибровочную степень свободы (репараметризации), во втором случае они имеют $(N+1)$ калибровочных степеней свободы, причем все связи являются квадратичными.

Мы показали, что в предложенной формулировке все первичные связи для систем с лагранжианами, линейно зависящими от внешних кривизн, сводятся к квадратичным, а алгоритм построения полного набора связей представляет собой набор алгебраических операций.

Система связей для модели с лагранжианом $F=c \tilde{k}_{N}$ оказалась әквивалентнойсистеме взаимодействующих $(N+1)$ осцилляторов, полученной дискретизацией струны $[11,12]$. Недавно Пашнев и Цулая провели БРСТ-квантование последней системы как для $N=1$ [13], так и для произвольного $N$ [14]. Мы полагаем, что такая удивительная параллель заслуживает более детального рассмотрения в отдельной работе. 
Благодарности. Автор считает своим долгом выразить благодарность Е. А. Иванову за предложение рассматривать системы с жесткостью, разъяснение их актуальности в контексте $W$-алгебр, полезные обсуждения и критику.

Многочисленные обсуждения были проведены с П. Пятовым и С. Ляховичем по процедуре Дирака, а по спиновым частицам - с С. Ляховичем, А. Шараповым и особенно К. Шехтером. А. Пашнев обратил внимание автора на соответствие между моделями жестких частиц и дискретных струн и указал на работы по БРСТ-квантованию последних. Завершению работы способствовал также постоянный интерес со стороны О. М. Худавердяна и В. В. Нестеренко.

Всем им автор выражает глубокую признательность.

Отдельно хочется поблагодарить И.В. Тютина, детально разъяснившего автору структуру калибровочных преобразований в формализме Дирака.

Работа выполнена при поддержке грантов INTAS-РФФИ № 95-0829 и INTAS-96-538, INTAS-93-127-ext.

\section{Список литературы}

[1] М. М. Постников. Лекции по геометрии. Семестр III: Гладкие многообразия. М.: Наука, 1987.

[2] A. M. Polyakov. Nucl. Phys. B. 1986. V. 268. P. 406; Mod. Phys. Lett. A. 1988. V. 3. P. 112.

[3] M.S. Plyushchay. Nucl. Phys. B. 1991. V. 362. P. 54; Yu. A. Kuznetsov, M.S. Plyushchay. Nucl. Phys. B. 1993. V. 389. P. 181.

[4] M.S. Plyushchay. Phys. Lett. B. 1990. V. 243. P. 383.

[5] M.S. Plyushchay. Phys. Lett. B. 1991. V. 253. P. 50.

[6] A. A. Kapustnikov, A. Pashnev, A. Pichugin. Phys. Rev. D. 1997. V. 55. P. 2257.

[7] E. Ramos, J. Roca. Nucl. Phys. B. 1995. V. 436. P. 529.

[8] E. Ramos, J. Roca. Nucl. Phys. B. 1995. V. 452. P. 705.

[9] И. В. Тютин. Частное сообшение. 1998.

[10] E. Ramos, J. Roca. Nucl. Phys. B. 1996. V. 477. P. 606.

[11] В. Д. Гериун, А. И. Пашнев. ТМФ. 1987. Т. 73. С. 294.

[12] A. P. Isaev, A. T. Filippov. Mod. Phys. Lett. A. 1989. V. 4. P. 2167.

[13] A. Pashnev, M. Tsulaia. Description of the higher massless irreducible integer spins in the BRST approach. hep-th/9803207; Препринт ОИЯИ Е2-98-56. Дубна: ОИЯИ, 1998.

[14] A. Pashnev, M. Tsulaia. In preparation. 